PROCEEDINGS OF THE

AMERICAN MATHEMATICAL SOCIETY

Volume 138, Number 11, November 2010, Pages 4129-4136

S 0002-9939(2010)10391-X

Article electronically published on May 27, 2010

\title{
THE TORSION INDEX OF A $p$-COMPACT GROUP
}

\author{
JAUME AGUADÉ
}

(Communicated by Brooke Shipley)

\begin{abstract}
We extend the theory of torsion indices of compact connected Lie groups to $p$-compact groups and compute these indices in all cases.
\end{abstract}

\section{INTRODUCTION AND STATEMENT OF RESULTS}

The torsion index of a compact connected Lie group was defined by Grothendieck in 1958 ([10]) and has been investigated by several authors ([14, 6], [15], etc.). Recently, the computation of the torsion indices of all simply connected compact Lie groups has been completed (see [16]). Since we are going to work at a single prime $p$, instead of the torsion index of a Lie group $G$, we want to consider its $p$ primary part $t_{p}(G)$. We summarize the properties of $t_{p}(G)$ which are relevant to the present work in the following proposition $\left(\mathbb{Z}_{p}\right.$ denotes the ring of $p$-adic integers).

Theorem 1.1. Let $p$ be a prime and let $G$ be a compact connected Lie group with a maximal torus $T$ and corresponding Weyl group $W$. The positive integer $t_{p}(G)$ has the following properties:

(TI1) If $A$ is a finite abelian p-subgroup of $G$, then $A$ has a subgroup of index dividing $t_{p}(G)$ which is contained in a conjugate of $T$.

(TI2) $t_{p}(G)$ kills the kernel and the cokernel of the homomorphism

$$
H^{*}\left(B G ; \mathbb{Z}_{p}\right) \rightarrow H^{*}\left(B T ; \mathbb{Z}_{p}\right)^{W}
$$

(TI3) $H^{*}\left(G / T ; \mathbb{Z}_{p}\right)$ is torsion free and concentrated in even degrees $\leq N=$ $\operatorname{dim}(G)-\operatorname{rank}(G)$, with $H^{N}\left(G / T ; \mathbb{Z}_{p}\right) \cong \mathbb{Z}_{p}$. Then, $t_{p}(G)$ is the order of the cokernel of $H^{N}\left(B T ; \mathbb{Z}_{p}\right) \rightarrow H^{N}\left(G / T ; \mathbb{Z}_{p}\right)$.

(TI4) If $p$ is not a torsion prime for $G$, then $t_{p}(G)=1$.

Notice that the property (TI3) can be taken as a definition of the ( $p$-primary) torsion index $t_{p}(G)$. The other properties are well known and can be found in 15], which provides proofs or references for all of them. Actually, the properties above are usually stated using $H^{*}(-; \mathbb{Z})$ and $t(G)=\prod_{p} t_{p}(G)$ instead of $H^{*}\left(-; \mathbb{Z}_{p}\right)$ and $t_{p}(G)$, but it is easy to see that both formulations are indeed equivalent. For property (TI2) one should notice that $H^{*}\left(B T ; \mathbb{Z}_{p}\right)^{W}=H^{*}(B T ; \mathbb{Z})^{W} \otimes \mathbb{Z}_{p}$. This follows from exactness of $-\otimes \mathbb{Z}_{p}$ and the fact that the elements invariant under $W$ can be viewed as the kernel of the homomorphism $\bigoplus_{g \in W}(1-g)$.

Received by the editors February 5, 2009.

2010 Mathematics Subject Classification. Primary 55P35, 57T15.

The author is partially supported by grants MTM2007-61545 and SGR2005-00606.

(C) 2010 American Mathematical Society Reverts to public domain 28 years from publication 
The purpose of this paper is to extend the theorem above to connected $p$-compact groups ([8]) and to compute the torsion indices in all cases. We prove:

Theorem 1.2. Let $p$ be a prime and let $X$ be a connected p-compact group with maximal torus $T$ and corresponding Weyl group $W$. There is an integer $t_{p}(X)$ such that:

(1) The properties (TI1), (TI2), (TI3), (TI4) in Theorem 1.1 hold after replacing $G$ with $X$.

(2) If $X$ is exotic, then $t_{p}(X)=1$ for $p$ odd and $t_{2}(X)=2$.

Here we use the work exotic with the same meaning as in [1]: A p-compact group $X$ is exotic if the associated pseudoreflection representation of the Weyl group of $X$ over the $p$-adic field is irreducible and does not come from a reflection group over $\mathbb{Z}$.

Section 2 deals with the (easier) odd prime case, and we show that if we define $t_{p}(X)=1$ for any exotic $X$, then properties (TI1), (TI2), (TI3), (TI4) hold true. The hardest part consists of computing the torsion index of the only exotic 2compact group, which we (following 12] denote $G_{3}$ (other authors denote it as $D I(4)$ ). We need a comprehensive review of the cohomology of $G_{3}$ and $B G_{3}$ (section (3) and some computations on the cohomology of the exotic homogeneous space $G_{3} / \operatorname{Spin}(7)$ (section (4) before we can prove that $t_{2}\left(G_{3}\right)=2$. Finally, we prove Theorem 1.2 in section 6 .

\section{THE ODD PRIME CASE}

The classification theorem for $p$-compact groups (2]) tells us that any connected $p$-compact group $X$ splits uniquely as a product $X \cong G_{p}^{\wedge} \times X_{1}$, where $G$ is a compact connected Lie group and $X_{1}$ is a product of exotic $p$-compact groups. Notice that the splitting is as $p$-compact groups and not just as spaces. This splitting implies that it is enough to prove Theorem 1.2 for each exotic $p$-compact group, since it is already known to be true for the ( $p$-completions of) compact connected Lie groups. Let us discuss this in some more detail. If Theorem 1.2 holds for the $p$-compact groups $X_{1}$ and $X_{2}$, let $X=X_{1} \times X_{2}$ and let us define $t_{p}(X)=t_{p}\left(X_{1}\right) t_{p}\left(X_{2}\right)$. We need to check that properties (TI1) to (TI4) hold for $X$ if they hold for $X_{1}$ and $X_{2}$. (TI4) is trivial and (TI3) is straightforward. To prove (TI2) let us observe that the kernel of $\gamma: H^{*}\left(B X ; \mathbb{Z}_{p}\right) \rightarrow H^{*}\left(B T ; \mathbb{Z}_{p}\right)^{W}$ is equal to the torsion elements in $H^{*}\left(B X ; \mathbb{Z}_{p}\right)$. If $X$ is of Lie type, this is well known (cf. [9]). If $X$ is exotic and $p=2$ (i.e. $X=G_{3}$ ), then this is assertion 4 in [12]; and if $p$ is odd, this is proven in [1. Then, it is clear that $t_{p}\left(X_{1}\right) t_{p}\left(X_{2}\right)$ kills the kernel of $\gamma$. It is obvious that $t_{p}\left(X_{1}\right) t_{p}\left(X_{2}\right)$ kills the cokernel of $\gamma$ as well. Finally, (TI1) follows easily since we can use the theory of kernels of homomorphisms between $p$-compact groups which is developed in [8], section 7 .

Let us assume now that $p$ is odd and let $X$ be an exotic $p$-compact group. These objects are very well understood. In particular, they satisfy the following properties (see [1]). Let $T$ and $W$ denote a maximal torus of $X$ and the corresponding Weyl group, respectively. Then:

(1) $X$ is simply connected and center free and $H^{*}\left(X ; \mathbb{Z}_{p}\right)$ is torsion free.

(2) The natural map $B T \rightarrow B X$ induces an isomorphism

$$
H^{*}\left(B X ; \mathbb{Z}_{p}\right) \cong H^{*}\left(B T ; \mathbb{Z}_{p}\right)^{W} .
$$


In particular, $H^{*}\left(B X ; \mathbb{Z}_{p}\right)$ is concentrated in even degrees.

(3) $H^{*}\left(X / T ; \mathbb{Z}_{p}\right)$ is a free $\mathbb{Z}_{p}$-module concentrated in even degrees. Moreover (see [13, th. 7.5.1) $H^{*}\left(X / T ; \mathbb{Z}_{p}\right) \otimes \mathbb{Q}$ is a Poincaré duality algebra with fundamental class in degree $\operatorname{dim}(X)-\operatorname{rank}(X)$. Actually, as a $W$-module, $H^{*}\left(X / T ; \mathbb{Z}_{p}\right) \otimes \mathbb{Q}$ coincides with the regular representation of $W$.

We also need another property of $p$-compact groups (which holds also for $p=2$ ) that follows from the work in [5].

(4) If $X$ is any $p$-compact group such that $H^{*}\left(B X ; \mathbb{F}_{p}\right)$ is concentrated in even degrees, then any finite abelian $p$-subgroup of $X$ is conjugated to a subgroup of the maximal torus of $X$. In particular, this holds for any product of exotic $p$-compact groups for $p$ odd.

Theorem 1.2 for $p$ odd follows immediately from all these properties of $p$-compact groups.

\section{The 2-COMPACt Group $G_{3}$ AND ITS MAXIMAL torus}

In this section we recollect several properties of $G_{3}$ that we need in the forthcoming sections. We state these properties without proof because either they can be found in the papers [7, 12, 4, [1] or they follow from straightforward computations that are left to the reader.

As is well known, $G_{3}$ is an exotic connected 2-compact group of rank three whose Weyl group $W$ is the reflection group number 24 in the Shephard-Todd list of finite complex reflection groups. Its existence was established by Dwyer and Wilkerson in [7]. We remind the reader that some authors call this 2-compact group $D I(4)$, but we follow the notation used in [12. As an abstract group, $W$ is isomorphic to $\mathbb{Z} / 2 \mathbb{Z} \times G L_{3}\left(\mathbb{F}_{2}\right)$ and for a maximal torus $T$ of $G_{3}$, there is a basis $\left\{\epsilon_{1}, \epsilon_{2}, \epsilon_{3}\right\}$ of $H^{2}\left(B T ; \mathbb{Z}_{2}\right)$ such that the action of $W$ on $H^{*}\left(B T ; \mathbb{Z}_{2}\right)$ is given by the pseudoreflections

$$
s_{1}=\left(\begin{array}{ccc}
-1 & -\bar{\alpha} & 1 \\
0 & 1 & 0 \\
0 & 0 & 1
\end{array}\right), \quad s_{2}=\left(\begin{array}{ccc}
1 & 0 & 0 \\
-\alpha & -1 & 1 \\
0 & 0 & 1
\end{array}\right), \quad s_{3}=\left(\begin{array}{ccc}
1 & 0 & 0 \\
0 & 1 & 0 \\
1 & 1 & -1
\end{array}\right),
$$

where $\alpha, \bar{\alpha} \in \mathbb{Z}_{2}$ are the roots of $x^{2}-x+2$ chosen in such a way that $\alpha$ is odd and $\bar{\alpha}$ is even.

$G_{3}$ has $\operatorname{Spin}(7)$ as a 2-compact subgroup of maximal rank. This means that there is a map $\phi: B \operatorname{Spin}(7)_{2}^{\wedge} \rightarrow B G_{3}$ whose homotopical fibre is $\mathbb{F}_{2}$-finite. It is natural to denote this fibre by $G_{3} / \operatorname{Spin}(7)$. The restriction of $\phi$ to a maximal torus of $\operatorname{Spin}(7)$ is a maximal torus of $G_{3}$.

There is a subgroup $V \subset \operatorname{Spin}(7)$ (explicitly described in [7]) which is an elementary abelian 2-group of rank four and such that the homomorphisms

$$
H^{*}\left(B G_{3} ; \mathbb{F}_{2}\right) \stackrel{\phi^{*}}{\longrightarrow} H^{*}\left(B \operatorname{Spin}(7) ; \mathbb{F}_{2}\right) \stackrel{k^{*}}{\longrightarrow} H^{*}\left(B V ; \mathbb{F}_{2}\right) \cong \mathbb{F}_{2}\left[V^{*}\right]
$$

are monomorphisms $\left(k^{*}\right.$ is induced by the inclusion $\left.V \subset \operatorname{Spin}(7)\right)$. Moreover, the image of $(\phi k)^{*}$ coincides with the rank four Dickson algebra which is the algebra of invariants of $H^{*}\left(B V ; \mathbb{F}_{2}\right)$ under the action of the full linear group $G L\left(V^{*}\right)$, and the image of $k^{*}$ coincides with the algebra of invariants $H^{*}\left(B V ; \mathbb{F}_{2}\right)^{H}$ where $H \subset G L\left(V^{*}\right)$ can be described, in some appropriate basis of $V^{*}$, as the set of matrices with first row equal to $(1,0,0,0)$. These algebras of invariants are well 
known (also as algebras over the Steenrod algebra) and we have isomorphisms (subscripts denote degrees)

$$
\begin{gathered}
H^{*}\left(B G_{3} ; \mathbb{F}_{2}\right) \cong \mathbb{F}_{2}\left[c_{8}, c_{12}, c_{14}, c_{15}\right], \\
H^{*}\left(B \operatorname{Spin}(7) ; \mathbb{F}_{2}\right) \cong \mathbb{F}_{2}\left[d_{4}, d_{6}, d_{7}, d_{8}\right],
\end{gathered}
$$

where the generators $c_{i}$ and $d_{i}$ can be explicitly described. In particular, we can see that $\phi^{*}$ is given by $\phi^{*}\left(c_{8}\right)=d_{4}^{2}+d_{8}, \phi^{*}\left(c_{12}\right)=d_{6}^{2}+d_{4} d_{8}, \phi^{*}\left(c_{14}\right)=d_{7}^{2}+d_{6} d_{8}$, $\phi^{*}\left(c_{15}\right)=d_{7} d_{8} . S q^{1}$ vanishes on $d_{4}, d_{7}, d_{8}$, while $S q^{1}\left(d_{6}\right)=d_{7}$.

As was said before, a maximal torus $T$ of $\operatorname{Spin}(7)$ is also a maximal torus of $G_{3}$. We have maps

$$
B T_{2}^{\wedge} \stackrel{i}{\rightarrow} B \operatorname{Spin}(7)_{2}^{\wedge} \stackrel{\phi}{\rightarrow} B G_{3}
$$

and we can view the Weyl group $W_{1}$ of $\operatorname{Spin}(7)$ as a subgroup of $W$, namely $W_{1}=$ $\left\langle s_{1}, s_{2}, s_{1} s_{3} s_{2} s_{1} s_{2} s_{3} s_{1}\right\rangle$. It is known that the homomorphism

$$
i^{*}: H^{*}\left(B \operatorname{Spin}(7) ; \mathbb{Z}_{2}\right) \rightarrow H^{*}\left(B T ; \mathbb{Z}_{2}\right)^{W_{1}}
$$

is surjective and its kernel coincides with the ideal of torsion elements. The integral invariants of $W_{1}$ are computed in 4. They turn out to form a polynomial algebra on generators of degrees $4,8,12$ :

$$
H^{*}\left(B T ; \mathbb{Z}_{2}\right)^{W_{1}} \cong \mathbb{Z}_{2}\left[u_{4}, u_{8}, u_{12}\right] .
$$

Choosing an appropriate basis $\left\{x_{1}, x_{2}, A\right\}$ of $H^{2}\left(B T ; \mathbb{Z}_{2}\right)$, these generators are

$$
\begin{aligned}
u_{4} & =(1 / 2)\left(x_{1}^{2}+x_{2}^{2}+x_{3}^{2}\right), \\
u_{8} & =(1 / 16)\left(x_{1}^{4}+x_{2}^{4}+x_{3}^{4}-2 x_{1}^{2} x_{2}^{2}-2 x_{1}^{2} x_{3}^{2}-2 x_{2}^{2} x_{3}^{2}\right), \\
u_{12} & =x_{1}^{2} x_{2}^{2} x_{3}^{2},
\end{aligned}
$$

where we have used the notation $x_{3}=2 A-x_{1}-x_{2}$, and one can check that in spite of the denominators, these polynomials belong to $\mathbb{Z}_{2}\left[x_{1}, x_{2}, A\right]$.

The generators $u_{4}, u_{8}$ and $u_{12}$ have a rather simple form as polynomials on $x_{1}, x_{2}, A$, but this basis of $H^{2}\left(B T ; \mathbb{Z}_{2}\right)$ does not coincide with the basis $\left\{\epsilon_{1}, \epsilon_{2}, \epsilon_{3}\right\}$ that we have used to describe the action of $W$ on $H^{*}\left(B T ; \mathbb{Z}_{2}\right)$. The matrix that expresses $\left\{\epsilon_{1}, \epsilon_{2}, \epsilon_{3}\right\}$ in terms of $\left\{x_{1}, x_{2}, A\right\}$ is

$$
\left(\begin{array}{ccc}
0 & -\bar{\alpha} / 2 & -(1+\alpha) / 2 \\
1 & 0 & -(1+\alpha) / 2 \\
0 & \bar{\alpha} & \alpha
\end{array}\right) \in G L_{3}\left(\mathbb{Z}_{2}\right) .
$$

Using this matrix we can express the generators $u_{4}, u_{8}, u_{12}$ as polynomials in $\epsilon_{1}, \epsilon_{2}$, $\epsilon_{3}$ and so we have an explicit description of the homomorphism

$$
\mathbb{Z}_{2}\left[u_{4}, u_{8}, u_{12}\right]=H^{*}\left(B \operatorname{Spin}(7) ; \mathbb{Z}_{2}\right) / \text { Torsion } \rightarrow H^{*}\left(B T ; \mathbb{Z}_{2}\right)=\mathbb{Z}_{2}\left[\epsilon_{1}, \epsilon_{2}, \epsilon_{3}\right] .
$$

Finally, we want to use this to describe the homomorphism

$$
\mathbb{F}_{2}\left[d_{4}, d_{6}, d_{7}, d_{8}\right]=H^{*}\left(B \operatorname{Spin}(7) ; \mathbb{F}_{2}\right) \stackrel{i^{*}}{\rightarrow} H^{*}\left(B T ; \mathbb{F}_{2}\right)=\mathbb{F}_{2}\left[\epsilon_{1}, \epsilon_{2}, \epsilon_{3}\right] .
$$

In the Bockstein spectral sequence for $B \operatorname{Spin}(7)$ we have $E_{2}=E_{\infty}=\mathbb{F}_{2}\left[\bar{d}_{4}, \bar{d}_{8}, \bar{d}_{6}^{2}\right]$, and the surjection

$$
j: H^{*}\left(B \operatorname{Spin}(7) ; \mathbb{Z}_{2}\right) / \text { Torsion } \rightarrow E_{\infty}
$$


is given by $j\left(u_{4}\right)=\bar{d}_{4}, j\left(u_{8}\right)=\bar{d}_{8}, j\left(u_{12}\right)=\bar{d}_{6}^{2}$. From this it is straightforward to perform the computations that yield

$$
\begin{aligned}
& i^{*}\left(d_{4}\right)=\epsilon_{1}^{2}+\epsilon_{1} \epsilon_{2}+\epsilon_{2}^{2}, \\
& i^{*}\left(d_{6}\right)=S q^{2} i^{*}\left(d_{4}\right)=\epsilon_{1}^{2} \epsilon_{2}+\epsilon_{1} \epsilon_{2}^{2}, \\
& i^{*}\left(d_{7}\right)=0, \\
& i^{*}\left(d_{8}\right)=\epsilon_{1} \epsilon_{2} \epsilon_{3}\left(\epsilon_{1}+\epsilon_{2}+\epsilon_{3}\right)+\epsilon_{3}^{2}\left(\epsilon_{1}+\epsilon_{2}+\epsilon_{3}\right)^{2} .
\end{aligned}
$$

\section{The exotic homogeneous SPaCe $G_{3} / \operatorname{Spin}(7)$}

In this section we want to investigate the cohomology of the exotic homogeneous space $G_{3} / \operatorname{Spin}(7)$. The computations presented here are probably known to experts, but it may be worthwhile to work them out here in some detail.

Let us consider the fibration $G_{3} / \operatorname{Spin}(7) \stackrel{\rho}{\rightarrow} B \operatorname{Spin}(7) \stackrel{\wedge}{\rightarrow} \rightarrow B G_{3}$ and let $V \subset$ Spin(7) denote the elementary abelian 2-group of rank 4 considered in the preceding section. To simplify the notation, let us write $S=H^{*}\left(B V ; \mathbb{F}_{2}\right)$. Then, we have $H^{*}\left(B \operatorname{Spin}(7) ; \mathbb{F}_{2}\right)=S^{H}$ and $H^{*}\left(B G_{3} ; \mathbb{F}_{2}\right)=S^{G}$ for $G=G L_{4}\left(\mathbb{F}_{2}\right)$.

The computation of $H^{*}\left(G_{3} / \operatorname{Spin}(7) ; \mathbb{F}_{2}\right)$ is best worked out with the EilenbergMoore spectral sequence

$$
\operatorname{Tor}_{H^{*}\left(B G_{3} ; \mathbb{F}_{2}\right)}\left(H^{*}\left(B \operatorname{Spin}(7) ; \mathbb{F}_{2}\right), \mathbb{F}_{2}\right) \quad \Rightarrow \quad H^{*}\left(G_{3} / \operatorname{Spin}(7) ; \mathbb{F}_{2}\right) .
$$

Here the key observation is that $H^{*}\left(B \operatorname{Spin}(7) ; \mathbb{F}_{2}\right)=S^{H}$ is a free module over $H^{*}\left(B G_{3} ; \mathbb{F}_{2}\right)=S^{G}$ because of the following classic argument. $S$ is an integral extension of $S^{G}$; hence $S^{H}$ is also an integral extension of $S^{G}$ and, since $S^{H}$ is a finitely generated algebra, we obtain that $S^{H}$ is a finitely generated $S^{G}$-module. But both $S^{H}$ and $S^{G}$ are polynomial algebras, and we can apply [3], Chap. V, 5.5, or [13, 6.7.1, to conclude that $S^{H}$ is $S^{G}$-free.

Hence the Eilenberg-Moore spectral sequence collapses to an isomorphism

$$
H^{*}\left(G_{3} / \operatorname{Spin}(7) ; \mathbb{F}_{2}\right) \cong \mathbb{F}_{2}\left[\bar{d}_{4}, \bar{d}_{6}, \bar{d}_{7}\right] /\left(\bar{d}_{6}{ }^{2}+{\overline{d_{4}}}_{4}{ }^{3}, \bar{d}_{7}{ }^{2}+\bar{d}_{4}{ }^{2} \bar{d}_{6}, \bar{d}_{4}{ }^{2} \bar{d}_{7}\right),
$$

where $\bar{d}_{4}, \bar{d}_{6}, \bar{d}_{7}$ are the images of $d_{4}, d_{6}, d_{7} \in H^{*}\left(B \operatorname{Spin}(7) ; \mathbb{F}_{2}\right)$, respectively. It is rather easy to completely work out the algebra structure of $H^{*}\left(G_{3} / \operatorname{Spin}(7) ; \mathbb{F}_{2}\right)$. We obtain the following:

(1) The Poincaré series of $H^{*}\left(G_{3} / \operatorname{Spin}(7) ; \mathbb{F}_{2}\right)$ is

$1+t^{4}+t^{6}+t^{7}+t^{8}+t^{10}+t^{11}+t^{12}+t^{13}+t^{14}+t^{16}+t^{17}+t^{18}+t^{20}+t^{24}$

and the Euler characteristic is $7=[W: H]$.

(2) An additive basis for $H^{*}\left(G_{3} / \operatorname{Spin}(7) ; \mathbb{F}_{2}\right)$ is given by

$$
\left\{\bar{d}_{4}{ }^{i}, i=0, \ldots, 6, \bar{d}_{6}, \bar{d}_{7}, \bar{d}_{4} \bar{d}_{6}, \bar{d}_{4} \bar{d}_{7}, \bar{d}_{6} \bar{d}_{7}, \bar{d}_{4}{ }^{2} \bar{d}_{6}, \bar{d}_{4} \bar{d}_{6} \bar{d}_{7}, \bar{d}_{4}{ }^{3} \bar{d}_{6}\right\} .
$$

(3) $H^{*}\left(G_{3} / \operatorname{Spin}(7) ; \mathbb{F}_{2}\right)$ is a Poincaré duality algebra with top class $\bar{d}_{4}{ }^{6}$ (see [13, 6.5).

(4) The Bockstein spectral sequence of $H^{*}\left(G_{3} / \operatorname{Spin}(7) ; \mathbb{F}_{2}\right)$ collapses after the second term; i.e. $H^{*}\left(G_{3} / \operatorname{Spin}(7) ; \mathbb{Z}_{2}\right)$ has only torsion of order 2 . We have

$$
H^{*}\left(G_{3} / \operatorname{Spin}(7) ; \mathbb{Z}_{2}\right) / \text { Torsion } \cong \mathbb{Z}_{2}[\bar{a}] / \bar{a}^{7}
$$

and

$$
H^{*}\left(G_{3} / \operatorname{Spin}(7) ; \mathbb{Z}_{2}\right) \cong \mathbb{Z}_{2}[\bar{a}, \bar{c}] /\left(\bar{a}^{7}, \bar{c}^{3}, \bar{a}^{2} \bar{c}, 2 \bar{c}\right)
$$


In particular, the top class in $H^{*}\left(G_{3} / \operatorname{Spin}(7) ; \mathbb{Z}_{2}\right)$ is $\bar{d}_{4}{ }^{6}$ in dimension 24 , and it is in the image of

$$
\phi^{*}: H^{*}\left(B \operatorname{Spin}(7) ; \mathbb{Z}_{2}\right) \rightarrow H^{*}\left(G_{3} / \operatorname{Spin}(7) ; \mathbb{Z}_{2}\right) .
$$

\section{The TORSION INDEX OF $G_{3}$}

To compute the torsion index of the 2-compact group $G_{3}$ we need a lemma on Poincaré duality in fibrations. I'm grateful to Aniceto Murillo for some helpful conversations on this subject. For this lemma we use the following notation. Let $\mathcal{O}$ denote the ring of integers or the ring of $p$-adic integers. Cohomology is taken with coefficients in $\mathcal{O}$, and we assume that all spaces are of finite type over $\mathcal{O}$. We say that $\eta \in H^{n}(X)$ is an orientation class if $H^{i}(X)=0$ for $i>n, H^{n}(X) \cong \mathcal{O}$, and $\eta$ is a generator of $H^{n}(X)$.

Lemma 5.1. Let $F \stackrel{j}{\rightarrow} E \stackrel{\pi}{\rightarrow} B$ be a fibration of 1-connected spaces and assume that $\eta^{F} \in H^{m}(F)$ and $\eta^{B} \in H^{n}(B)$ are orientation classes. Assume $\alpha \in H^{m}(E)$ is such that $j^{*}(\alpha)=\lambda \eta^{F}$ for some $\lambda \neq 0$. Then there is an orientation class $\eta^{E}$ for $E$ such that $\alpha \cdot \pi^{*}\left(\eta^{B}\right)=\lambda \eta^{E}$.

Proof. This follows easily from the cohomology spectral sequence of the fibration $F \stackrel{j}{\rightarrow} E \stackrel{\pi}{\rightarrow} B$. First of all, it is clear that $H^{i}(E)=0$ for $i>n+m$ while $H^{n+m}(E)=E_{\infty}^{n, m}=E_{2}^{n, m} \cong \mathcal{O}$. Recall that the cohomology spectral sequence is multiplicative in the sense that (up to some signs which would not play any role here) the product in $E_{2}$ induced by the products in $H^{*}(B)$ and $H^{*}(F)$ yields a product in each $E_{r}, 2 \leq r \leq \infty$, in such a way that the product in $E_{\infty}$ is compatible with the product in $H^{*}(E)$.

At the $E_{2}$ level we have that $\eta^{E}:=\eta^{F} \cdot \eta^{B}$ is a generator of $E_{2}^{n, m}=E_{\infty}^{n, m}=$ $H^{n+m}(E)$. The hypothesis $j^{*}(\alpha)=\lambda \eta^{F}, \lambda \neq 0$ implies that $\alpha$ has filtration zero in $H^{m}(E)$ and its image in $E_{\infty}^{0, m}$ is $\lambda \eta^{F}$. Then, $\lambda \eta^{E}=\left(\lambda \eta^{F}\right) \cdot\left[\eta^{B}\right]$ holds in $E_{\infty}$ where $\left[\eta^{B}\right]$ denotes the image of $\eta^{B}$ in $E_{\infty}^{n, 0}$. Since $E_{\infty}^{i, m+n-i}=0$ for $i \neq n$, we deduce $\lambda \eta^{E}=\alpha \cdot \pi^{*}\left(\eta^{B}\right)$, as desired.

Now we can proceed to the computation of the torsion index of $G_{3}$ or, to be more precise, to the computation of the order of the cokernel of $k^{*}: H^{42}\left(B T ; \mathbb{Z}_{2}\right) \rightarrow$ $H^{42}\left(G_{3} / T ; \mathbb{Z}_{2}\right)$. We consider the diagram

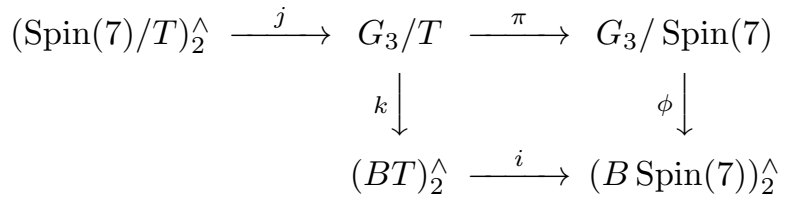

$\operatorname{Spin}(7) / T$ is a compact orientable differentiable manifold of dimension 18 , and we can choose an orientation class $\eta \in H^{18}\left(\operatorname{Spin}(7) / T ; \mathbb{Z}_{2}\right)$. The torsion indices of the Lie groups $\operatorname{Spin}(n)$ have been computed by Totaro for all values of $n([15])$, and it turns out that the torsion index of $\operatorname{Spin}(7)$ is equal to 2 . This means that there is $\omega \in H^{*}(B T ; \mathbb{Z})$ such that $f^{*}(\omega)=2 \eta$ for the natural map $f: \operatorname{Spin}(7) / T \rightarrow B T$.

The computations in the preceding section show that there is an orientation class $\rho \in H^{24}\left(G_{3} / \operatorname{Spin}(7) ; \mathbb{Z}_{2}\right)$ which is in the image of $\phi^{*}$. Let $\rho=\phi^{*}(\gamma)$. We can now apply the lemma above to the fibration $\operatorname{Spin}(7) / T \rightarrow G_{3} / T \rightarrow G_{3} / \operatorname{Spin}(7)$ with $\alpha=k^{*}(\omega)$ and deduce that there is an orientation class $\theta \in H^{42}\left(G_{3} / T ; \mathbb{Z}_{2}\right)$ such that $k^{*}\left(\omega \cdot i^{*}(\gamma)\right)=2 \theta$. This implies that the torsion index of $G_{3}$ divides 2 . 
Next, we prove that the torsion index of $G_{3}$ cannot be equal to 1 . It is enough to prove that the homomorphism $H^{42}\left(B T ; \mathbb{F}_{2}\right) \rightarrow H^{42}\left(G_{3} / T ; \mathbb{F}_{2}\right)$ is equal to zero. Let us consider the $\mathbb{F}_{2}$-spectral sequence of the fibration $G_{3} \rightarrow G_{3} / T \rightarrow B T_{2}^{\wedge}$. We have that

$$
\begin{gathered}
H^{*}\left(G_{3} ; \mathbb{F}_{2}\right) \cong \mathbb{F}_{2}\left[x_{7}\right] / x_{7}^{4} \otimes E\left(x_{11}, x_{13}\right), \\
S q^{4}\left(x_{7}\right)=x_{11}, S q^{2}\left(x_{11}\right)=x_{13}, S q^{1}\left(y_{13}\right)=x_{7}^{2} .
\end{gathered}
$$

Hence, the generators $x_{7}, x_{11}, x_{13}, x_{7}^{2}$ are transgressive to $c_{8}, c_{12}, c_{14}, 0$, respectively. Here we denote by $c_{8}, c_{12}, c_{14}$ the images in $H^{*}\left(B T ; \mathbb{F}_{2}\right)$ of the generators $c_{8}, c_{12}, c_{14}$ $\in H^{*}\left(B G_{3} ; \mathbb{F}_{2}\right)$. Recall that in section 3 we have computed these elements as explicit polynomials in some basis $\left\{\epsilon_{1}, \epsilon_{2}, \epsilon_{3}\right\}$ of $H^{2}\left(B T ; \mathbb{F}_{2}\right)$.

In the $E_{2}$-term of the spectral sequence of $G_{3} \rightarrow G_{3} / T \rightarrow B T_{2}^{\wedge}$, let us consider the row containing $x_{7}^{2}$. All elements in this row are permanent cycles, and the only boundaries are the elements of the form $x_{7}^{2} q$ with $q$ in the ideal of $\mathbb{F}_{2}\left[\epsilon_{1}, \epsilon_{2}, \epsilon_{3}\right]$ generated by $c_{8}, c_{12}, c_{14}$. If we compute the quotient algebra $\mathbb{F}_{2}\left[\epsilon_{1}, \epsilon_{2}, \epsilon_{3}\right] /\left(c_{8}, c_{12}, c_{14}\right)$ (using any choice of computer algebra software), we see that it is a graded algebra with Poincaré series equal to

$$
\begin{aligned}
1+3 t^{2}+6 t^{4}+10 t^{6}+14 t^{8} & +18 t^{10}+21 t^{12}+22 t^{14} \\
+ & 21 t^{16}+18 t^{18}+14 t^{20}+10 t^{22}+6 t^{24}+3 t^{26}+t^{28},
\end{aligned}
$$

and so in particular there is an element $q \in H^{28}\left(B T ; \mathbb{F}_{2}\right)$ which does not belong to the ideal $\left(c_{8}, c_{12}, c_{14}\right)$. Hence, the element $x_{7}^{2} q$ in the $E_{2}$-term of the spectral sequence survives to a nontrivial element in $H^{42}\left(G_{3} / T ; \mathbb{F}_{2}\right)$ which cannot be in the image of $H^{*}\left(B T ; \mathbb{F}_{2}\right)$. This finishes the proof of

Theorem 5.2. The cokernel of $H^{42}\left(B T ; \mathbb{Z}_{2}\right) \rightarrow H^{42}\left(G_{3} / T ; \mathbb{Z}_{2}\right)$ has order two.

\section{Proof of Theorem 1.2}

In section 2 we saw that it is enough to prove Theorem 1.2 for each exotic $p$-compact group, and we also saw that Theorem 1.2 is true for all odd primes. Since it is known (2]) that the only exotic 2-compact group is $G_{3}$, the only thing that remains to be proved is that $G_{3}$ satisfies the properties (TI1) to (TI4) with $t_{2}\left(G_{3}\right)=2$.

(TI4) is void, and (TI3) is just Theorem 5.2 plus some facts about $G_{3} / T$ which were proven in $\left[2\right.$. In $\left[12\right.$ it is proven that the torsion elements in $H^{*}\left(B G_{3} ; \mathbb{Z}_{2}\right)$ are of order two and the homomorphism $H^{*}\left(B G_{3} ; \mathbb{Z}_{2}\right) \rightarrow H^{*}\left(B T ; \mathbb{Z}_{2}\right)^{W}$ is surjective. This implies immediately that (TI2) holds. Let $A$ be a nontrivial finite abelian 2-subgroup of $G_{3}$ and let $E$ be a subgroup of $A$ of order 2. Then, $A$ factors through the centralizer of $E$ in $G_{3}$ which is $\operatorname{Spin}(7)$. Since $\operatorname{Spin}(7)$ has 2-torsion index equal to 2 , we deduce that $A$ has a subgroup of index at most 2 which is included in a maximal torus of $G_{3}$. So, we have (TI1), and the proof is complete.

\section{REFERENCES}

1. K.K.S. Andersen, J. Grodal, J.M. Møller, A. Viruel, The classification of p-compact groups for $p$ odd, Ann. of Math. (2) 167 (2008), 95-210. MR2373153 (2009a:55012)

2. K.K.S. Andersen, J. Grodal, The classification of 2-compact groups, J. Amer. Math. Soc. 22 (2009), no. 2, 387-436. MR2476779

3. N. Bourbaki, Groupes et algèbres de Lie, Ch. 4, 5 et 6. Act. Sci. Ind. 1337, Paris, 1968. MR 0240238 (39:1590) 
4. D.J. Benson, J.A. Wood, Integral invariants and cohomology of B $\operatorname{Spin}(n)$, Topology 34 (1995), 13-28. MR:1308487(95m:55023)

5. F.-X. Dehon, J. Lannes, Sur les espaces fonctionnels dont la source est le classifiant d'un groupe de Lie compact commutatif, Inst. Hautes Études Sci. Publ. Math., No. 89 (1999), 127-177. MR:1793415 (2001m:55038)

6. M. Demazure, Invariants symétriques entiers des groupes de Weyl et torsion, Invent. Math. 21 (1973), 287-301. MR0342522 (49:7268)

7. W. G. Dwyer, C. W. Wilkerson, A new finite loop space at the prime 2, J. Amer. Math. Soc. 6 (1993), 37-64. MR1161306 (93d:55011)

8. W. G. Dwyer, C. W. Wilkerson, Homotopy fixed point methods for Lie groups and finite loop spaces, Ann. of Math. (2) 139 (1994), 395-442. MR1274096 (95e:55019)

9. M. Feshbach, The image of $H^{*}(B G ; \mathbb{Z})$ in $H^{*}(B T ; \mathbb{Z})$ for $G$ a compact Lie group with maximal torus T, Topology 20 (1981), 93-95. MR592571 (82g:55019)

10. A. Grothendieck, La torsion homologique et les sections rationnelles, in Anneaux de Chow et applications, Séminaire C. Chevalley, 1958, 2nd year, Secrétariat math., Paris, exp. 5.

11. D. Notbohm, On the 2-compact group DI(4), J. Reine Angew. Math. 555 (2003), 163-185. MR.1956596 (2003k:55018)

12. A. Osse, U. Suter, Invariant theory and the K-theory of the Dwyer-Wilkerson space, Contemp. Math., 265, Amer. Math. Soc., Providence, RI, 2000, 17-185. MR1803957 (2001j:55005)

13. L. Smith, Polynomial Invariants of Finite Groups. A K Peters, Wellesley, Mass., 1995. MR.1328644 (96f:13008)

14. J. Tits, Sur les degrés des extensions de corps déployant les groupes algébriques simples, C. R. Acad. Sci. Paris Ser. I. Math. 315 (1992), 1131-1138. MR1194504 (93m:20059)

15. B. Totaro, The torsion index of the spin groups, Duke Math. J. 129 (2005), 249-290. MR2165543 (2006f:57039b)

16. B. Totaro, The torsion index of $E_{8}$ and other groups, Duke Math. J. 129 (2005), 219-248. MR 2165542(2006f:57039a)

Departament de Matemàtiques, Universitat Autònoma de Barcelona, 08193 CerdaNYOLA DEL VALLÈs, SPAiN

E-mail address: aguade@mat.uab.cat 